\title{
EDS Assisted by Precessing Electron Beam
}

\author{
Yifeng Liao and Laurence D. Marks \\ Department of Materials Science and Engineering, Northwestern University, Evanston, IL USA
}

EDS has been widely used for determining local chemical composition. The accuracy can be about several percent in well-structured experiments. In practice, however, the measurement is largely influenced by the sample orientation, and electron channeling effects should be avoided for most conventional measurements. In this study, we employ precession electron beam to EDS measurements in a TEM. It is demonstrated that by precessing the electron beam at a large angle, the electron channeling effects can be significantly reduced [1].

A (001)-oriented $\mathrm{SrTiO}_{3}$ specimen and an $\mathrm{L}_{1}$-ordered intermetallic specimen with nominal composition $\mathrm{Fe}_{55} \mathrm{Mn}_{19} \mathrm{Al}_{26}$ were examined. The oxide specimen was prepared using conventional ion milling, while the intermetallic specimen was electropolished in $30 \%$ nitric acid at $253 \mathrm{~K}$. A JEOL 2100F field emission TEM operated at $200 \mathrm{kV}$ was employed. The TEM is equipped with a Bruker EDS system, a Gatan EELS system, and a home-built precession electron diffraction (PED) system [2]. The PED system was aligned using the method described previously in order to stabilize the illumination to a fixed area [3]. The specimens were tilted to either zone axis or near two beam conditions where the electron channeling is enhanced.

Fig. 1a shows normalized EDS spectra which were obtained with and without precession electron beam. In the conventional EDS measurement for the $\mathrm{SrTiO}_{3}$ oriented along the [001] zone axis, the $\mathrm{Sr} / \mathrm{Ti}$ ratio was measured to be $0.75-0.80$. The $\mathrm{Sr} / \mathrm{Ti}$ ratio significantly increased when the beam was precessed at an angle of $33.92 \mathrm{mRad}$. The reduction of the electron channeling effects is dependent on the precession angle. When the electron beam was precessed at an angle of 5 $\mathrm{mRad}$, the $\mathrm{Sr} / \mathrm{Ti}$ was 0.79 . The $\mathrm{Sr} / \mathrm{Ti}$ ratio increased remarkably with increasing the precession angle, and reached a plateau of 0.99 for precession angle greater than $22.54 \mathrm{mRad}$, see Fig. $1 \mathrm{~b}$. The intensities of the EDS signals decreased by $\sim 20 \%$ when the electrons beam precessed at an angle of $33.92 \mathrm{mRad}$. The influence of PED was subsequently examined in a series of ALCHEMI-like experiments in which the $\mathrm{SrTiO}_{3}$ specimen was titled to near two-beam conditions with different deviation $\mathrm{s}_{\mathrm{z}}$ to the Bragg condition $\mathrm{g}=(200)$, see Fig.2a. The Sr/Ti ratio increased from 0.93 to 1.20 when $\mathrm{s}_{\mathrm{z}}$ changed from negative to positive which is expected in such experiments. For the PED-assisted experiments, the Sr/Ti ratio was measured to be $\sim 1.04$ and was insensitive to the deviation.

The reduction of electron channeling effect by PED is verified using the intermetallic specimen. The measured composition using conventional EDS had a lower Fe concentration (55\%) compared to the off-zone-axis measurement of $58.8 \% \pm 1.7 \%$, while this difference was eliminated for the PED-assisted EDS measurement when the precession angle was greater than $47.48 \mathrm{mRad}$. As shown in Fig. 2b, the Fe composition in the ALCHEMI-like experiments $(\mathrm{g}=(200))$ varied between $55 \%$ and $63 \%$ for EDS experiments, and remained $\sim 58 \%$ for all PEDEDS experiments. It is clear that the channeling effects in EDS measurements are effectively reduced at a large precession angle. 
References:

[1] Y. Liao and L.D. Marks, Ultramicroscopy, 126 (2013), p. 19-22.

[2] C.S. Own, L.D. Marks, and W. Sinkler, Review of Scientific Instruments, 76 (2005), p. 033703 1-13.

[3] Y. Liao and L.D. Marks, Ultramicroscopy, 117 (2012), p. 1-6.

[4] The authors acknowledge funding from the NSF on grant number CMMI-1030703 and by the NIH on grant number 1RC2AR058993-01.
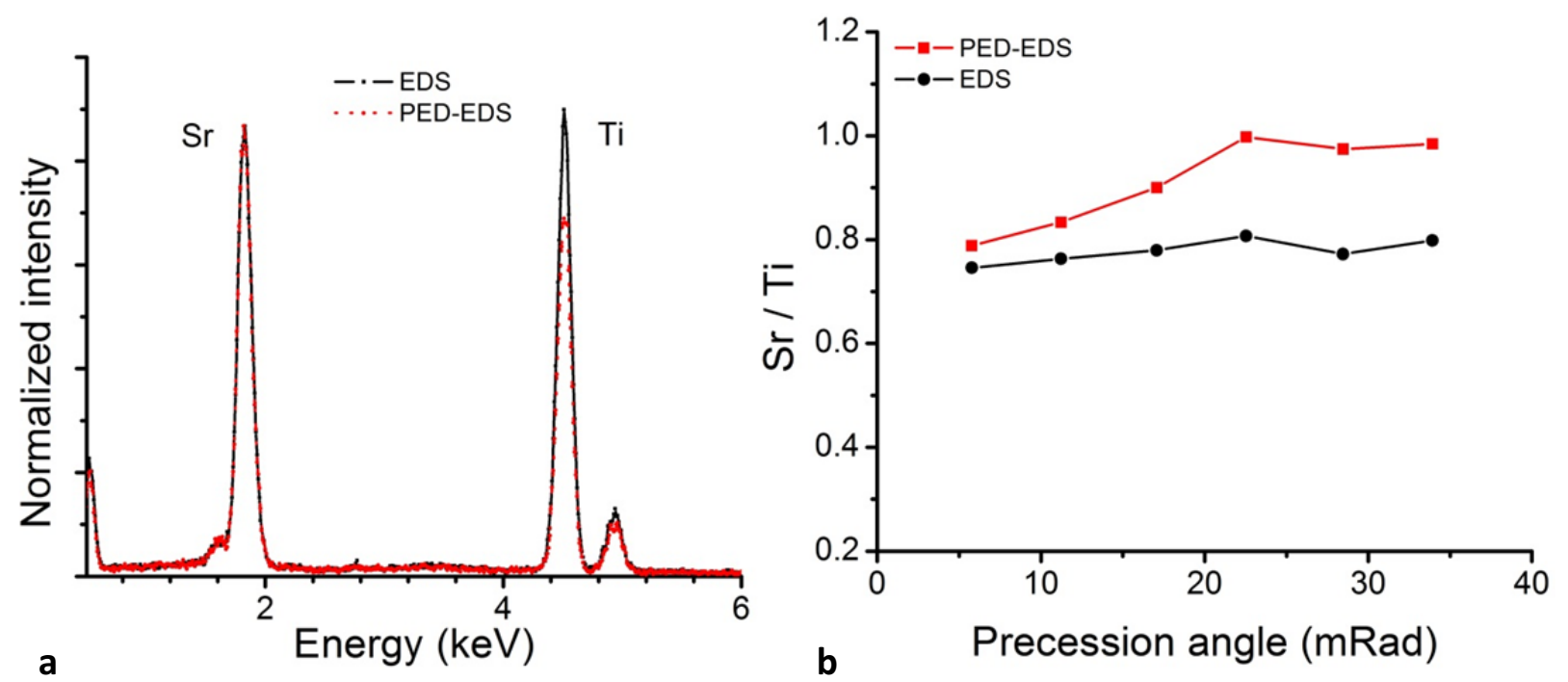

Figure 1: (a) Normalized EDS spectra of $\mathrm{SrTiO}_{3}$ at the [001] zone axis. The precession angle was $33.92 \mathrm{mRad}$. (b) $\mathrm{Sr} / \mathrm{Ti}$ ratio as a function of precession angle.
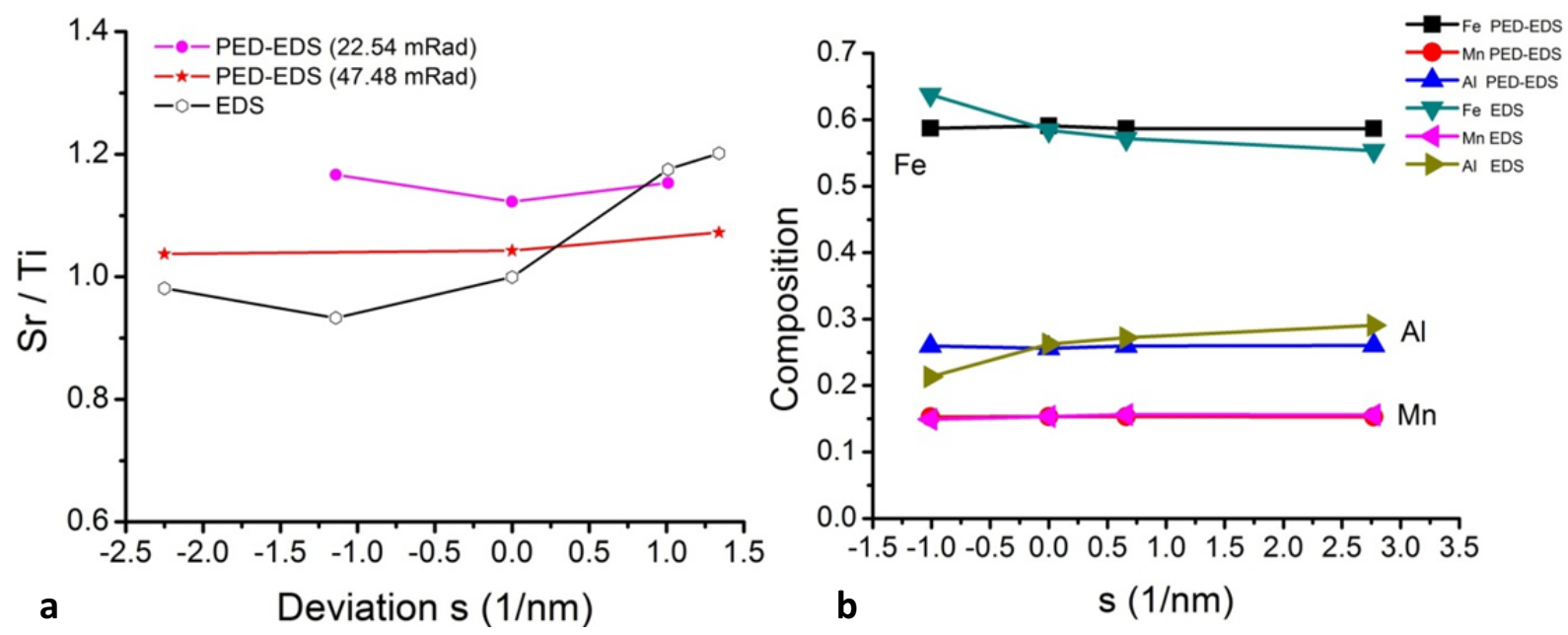

Figure 2: EDS and PED-EDS m easurements in the ALCHEMI-like experiments. (a) Sr/Ti ratio as a function of deviation fro $\mathrm{m}$ the two-beam condition. The precession angles were 22.54 and 47.48 mRad. (b) The com position of the Fe-Mn-Al alloy as a fun ction of deviation. The precession angle was set to $47.48 \mathrm{mRad}$. 